\title{
LIX. A catalogue of comets
}

\section{Rev. T.J. Hussey A.M.}

To cite this article: Rev. T.J. Hussey A.M. (1834) LIX. A catalogue of comets, Philosophical Magazine Series 3, 4:23, 349-352, DOI: 10.1080/14786443408648352

To link to this article: http://dx.doi.org/10.1080/14786443408648352

册 Published online: 01 Jun 2009.

Submit your article to this journal 준

LII Article views: 2

Q View related articles $₫$ 


\section{[ $\quad 349$ ]}

LIX. A Catalogue of Comets. By the Rev. T. J.Hussey, A.M., Rector of Hayes, Kent.

[Continued from p. 207.]

[The Chronology employed is that of Petau or Petavius.]

A, the comet of 1680 . B, that of 1652. C (Halley's), that of 1682. D, that of $1759 . \quad \mathrm{E}$, that of 1661 . F, that of 1677. G, that of 1556 . H, that of 1665 . I, that of 1585 . $\mathrm{K}$, that of $1744 . \quad \mathrm{L}$, that of 1737 .

\begin{tabular}{|c|c|c|c|c|c|c|}
\hline 它 & $\begin{array}{c}\text { Year of } \\
\text { Appear- } \\
\text { ance } \\
\text { A. C. }\end{array}$ & $\begin{array}{l}\text { Same } \\
\text { as that } \\
\text { of }\end{array}$ & $\begin{array}{l}\text { Month or Season } \\
\text { when it agpeared. }\end{array}$ & $\begin{array}{c}\text { Place or Direction } \\
\text { in whichl it ap- } \\
\text { peared. }\end{array}$ & $\begin{array}{l}\text { By whom men- } \\
\text { tioned. }\end{array}$ & Remarks. \\
\hline & 1338 & $\cdots$ & April & Gemini & Chr.Ro & \\
\hline 53 & 1340 & ... & Febr., March.. & Scor., Vir, Leo & Ch.Rec.,Gregor., & \\
\hline 354 & 1345 & $\ldots$ & July & Ur. Maj., Leo.. & Gregora Byz. & \\
\hline & 1347 & & August. & CanurMeduse & Hist. & \\
\hline & $104 /$ & $\cdots$ & Jaghose. & Capulineuste & $\begin{array}{l}\text { Giov. Viliani. } \\
\text { Gion. }\end{array}$ & \\
\hline 356 & 1351 & $\cdots$ & Nov., Dec..... & $\begin{array}{l}\text { Andro, Aries, } \\
\text { Taurus, Canc. }\end{array}$ & Chin. Rec., Matt. & \\
\hline 357 & 1356 & ... & Sept.Oct.Nov. & & $\begin{array}{l}\text { Villani, \&c.\&c. } \\
\text { Chinese Records }\end{array}$ & $\begin{array}{l}\text { Elementscom- } \\
\text { puted by }\end{array}$ \\
\hline 358 & 1360 & $\cdots$ & March .. & Tow d $^{3}$ the east & $\begin{array}{l}\text { Chin.Rec., Chro. } \\
\text { Z }\end{array}$ & Burckbardt*. \\
\hline 359 & 1362 & ... & June,July,Aug. & $\begin{array}{c}\text { Aquar., Pegas., } \\
\text { Taurus..... } \\
\text { Capricornus... }\end{array}$ & $\begin{array}{l}\text { Ch. Rec., Syn. } \\
\text { Chr.Nangis,\&c. } \\
\text { Chinese Records. }\end{array}$ & $\begin{array}{l}\text { Elements com- } \\
\text { puted by } \\
\text { Burckhardt*. }\end{array}$ \\
\hline 361 & 1363 & $\ldots$ & March.............. & & Chinese Records. & Seen for a \\
\hline$|362|$ & 1366 & $\ldots$ & August ........ & $\begin{array}{r}\text { Ur.Maj, Scor- } \\
\text { pio, Aquarius }\end{array}$ & & month \\
\hline 363 & 1368 & $\cdots$ & $\begin{array}{r}\text { Feb., March, } \\
\text { April ...... }\end{array}$ & Taurt & Chin. & \\
\hline 364 & 1371 & ... & January......... & $\begin{array}{r}\text { Towards the } \\
\text { north ......... }\end{array}$ & $\begin{array}{l}\text { singh., Nan. \&c. } \\
\text { Bonincont. Ann. }\end{array}$ & \\
\hline 365 & 1376 & $\dddot{0}$ & June & & Chinese Records. & \\
\hline 366 & 1378 & $\mathbf{B}$ & Sep & $\begin{array}{r}\text { Antinous, Ur. } \\
\text { Major ....... }\end{array}$ & Chin & Motion very \\
\hline 367 & 1380 & $\mathrm{C}$ & November..... & .. & $\begin{array}{c}\text { Chi.Rec., Chron. } \\
\text { Citizense. }\end{array}$ & $\begin{array}{l}\text { trograde. } \\
\text { tre- }\end{array}$ \\
\hline 368 & $138^{2}$ & $\ldots$ & March .... & $\ldots .$. & $\begin{array}{l}\text { Chi.Rec., Chron. } \\
\text { Bothon. }\end{array}$ & \\
\hline 36 & - & $\ldots$ & August... & $\ldots$ & $\begin{array}{l}\text { Annal. Aug. Bon- } \\
\text { fin. Ann. }\end{array}$ & \\
\hline 370 & - & $\ldots$ & December...... & $\begin{array}{l}\text { To the west- } \\
\text { ward }\end{array}$ & Non & \\
\hline 371 & 1385 & $\cdots$ & October & Ur.Maj.,Hyd., & & \\
\hline$|372|$ & 1391 & $\cdots$ & May & Ursa Major... & $\begin{array}{r}\text { Ann.Foroliv.,Ch. } \\
\text { Records ....... }\end{array}$ & Small \& faint. \\
\hline
\end{tabular}

* See note, page 352. 
Rev. T. J. Hussey's Catalogue of Comets.

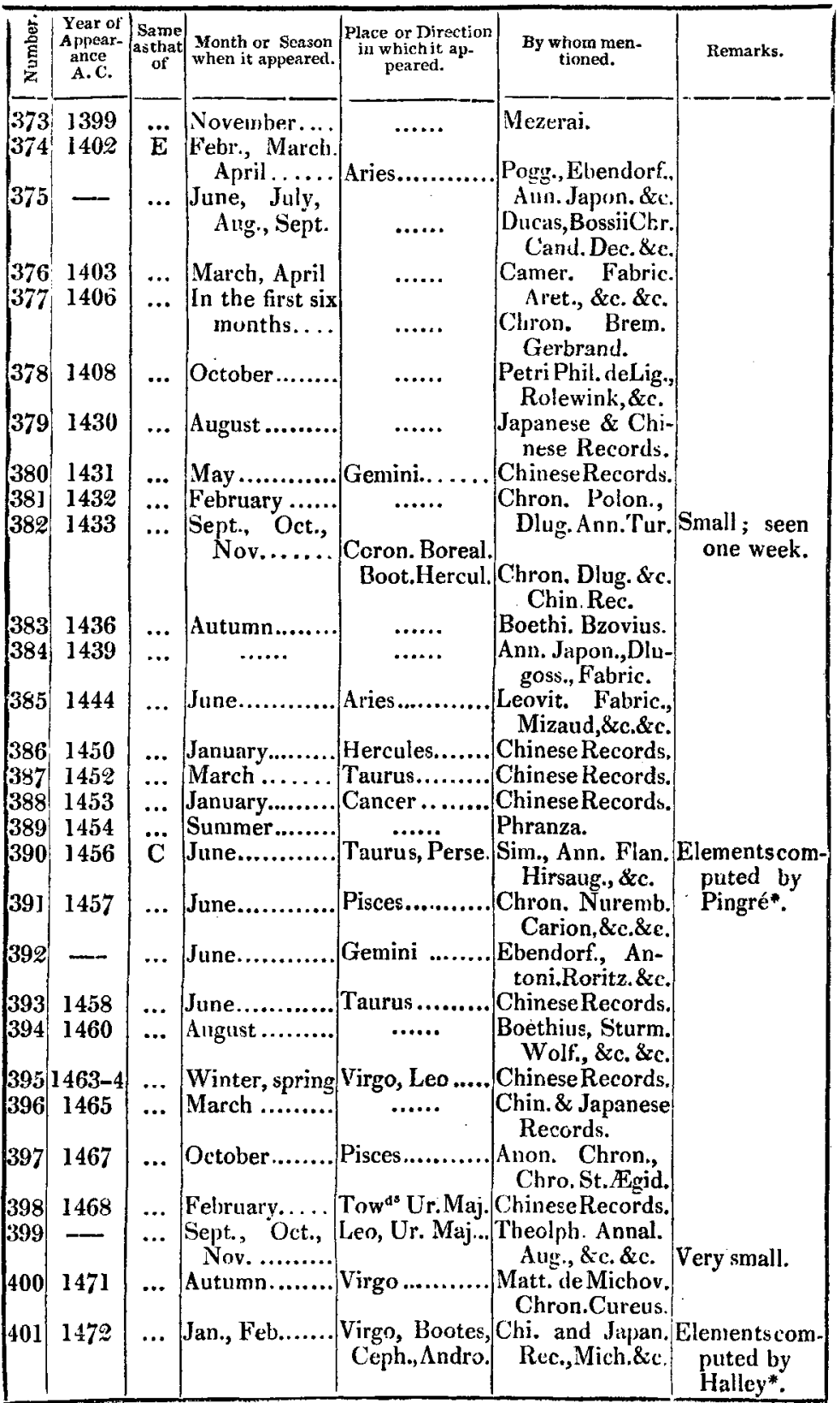

* See note, page $35 \%$. 
Rev. T. J. Hussey's Catalogue of Comets.

\begin{tabular}{|c|c|c|c|c|c|c|}
\hline 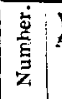 & $\begin{array}{l}\text { Year of } \\
\text { Appear- } \\
\text { ance- } \\
\text { A.C. }\end{array}$ & $\begin{array}{l}\text { Same } \\
\text { as that } \\
\text { of }\end{array}$ & $\begin{array}{l}\text { Month or Season } \\
\text { when it appeared. }\end{array}$ & $\mid \begin{array}{c}\text { Place or Direction } \\
\text { in which it ap- } \\
\text { peared. }\end{array}$ & $\begin{array}{l}\text { By whom men- } \\
\text { tioned. }\end{array}$ & Remarks. \\
\hline 4021 & $1476-7$ & $\cdots$ & Dec., Jan...... & ...... & Ripamont. Pon- & $t_{0}$ \\
\hline 403 & 1477 & $\cdots$ & December.... & & Chron. Bossian. & smal. \\
\hline 404 & 1478 & ... & September..... & $\cdots \cdots$ & $\begin{array}{c}\text { Chron. Bossian., } \\
\text { Cavitellius. }\end{array}$ & \\
\hline 405 & 1479 & $\cdots$ & $\cdots \cdots$ & $\cdots \cdots$ & $\begin{array}{l}\text { Lycosthenes, He. } \\
\text { velius. }\end{array}$ & \\
\hline 406 & 1491 & $\cdots$ & Jan., Feb.. & Pisces, Aries. & $\begin{array}{l}\text { Chron. Boss., Mi- } \\
\text { chov., Region. }\end{array}$ & \\
\hline 407 & $\int ?$ & $\cdots$ & January & Cygnus.. & Chinese Records. & \\
\hline 408 & $\left\{\begin{array}{l}1494 \\
\text { or- } 5\end{array}\right.$ & $\cdots$ & ...... & ...... & Scaliger. & \\
\hline 409 & $\begin{array}{l}(0 r-b \\
1500\end{array}$ & $\cdots$ & April, May..... & Sagit., Capric. & $\begin{array}{c}\text { Ch.Rec, Michov. } \\
\text { Comiers, \&c. }\end{array}$ & \\
\hline 410 & 1503 & $\cdots$ & August....... & ....... & $\begin{array}{l}\text { Chron. Waldsas- } \\
\text { sense. }\end{array}$ & \\
\hline 411 & 1505 & $\cdots$ & April. & Aries......... & Chron. German., & \\
\hline 412 & 1506 & ... & July, August. . & Near the Pole & Chin. and Japan. & \\
\hline 413 & 1512 & $\cdots$ & March, April... & $\cdots \cdots$ & Chron. Magd., & \\
\hline 414 & $1513-4$ & $\cdots$ & Dec.,Jan., Feb. & Can.,Leo,Virg. & Vicom., Schuler. & \\
\hline 415 & 1516 & ... & January.......... & & $\begin{array}{r}\text { Bizar.Gen. Hist., } \\
\text { Chr.Mlariff.\&rc. }\end{array}$ & - Seen only a \\
\hline 416 & 1518 & $\cdots$ & April & ...... & Cavitellius. & \\
\hline 417 & 1521 & ... & June. & $\cdots \cdots$ & $\begin{array}{l}\text { Keckerman, } \mathrm{Ca}- \\
\text { vitellius, \&c. }\end{array}$ & \\
\hline 418 & 1522 & $\cdots$ & & ....... & $\begin{array}{l}\text { Mizaud.Rockem- } \\
\text { bach, \&c. }\end{array}$ & \\
\hline 419 & 1523 & $\cdots$ & October ........ & $\ldots \ldots$ & $\begin{array}{l}\text { Schuler, Rockem- } \\
\text { bach, dzc. }\end{array}$ & \\
\hline 420 & 1528 & $\cdots$ & Januar & ...... & Littara, Riccioli. & \\
\hline 421 & 1530 & $\ldots$ & Novem & $\cdots \cdots$ & $\begin{array}{l}\text { Mizald. Cardan., } \\
\text { \&c. \&c. }\end{array}$ & \\
\hline 422 & 1531 & $\mathbf{C}$ & July, August... & Gemini & $\begin{array}{l}\text { Chin. and Japan. } \\
\text { Rec., Vapov., } \\
\text { Guicciard.\&c. }\end{array}$ & $\begin{array}{l}\text { Elementscom- } \\
\text { puted by } \\
\text { Halley. }\end{array}$ \\
\hline 423 & 1532 & $\mathrm{E}$ & Sep.,Oct.,Nov. & v. Virgo, Libra. & 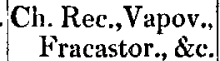 & $\begin{array}{l}\text { Elements com- } \\
\text { puted as in }\end{array}$ \\
\hline 424 & 1533 & $\cdots$ & June,July, Ang. & $\begin{array}{r}\text { Gemi., Taurus, } \\
\text { Aries ....... }\end{array}$ & Ch.Rec., Gassar. & $\begin{array}{l}\text { note* } \\
\text { Elements com- }\end{array}$ \\
\hline 425 & 1538 & K & Januar & Pisces & $\begin{array}{l}\text { Ann.Vapo. \&c. } \\
\text { Eher. Mizald., }\end{array}$ & $\begin{array}{l}\text { puted as be- } \\
\text { low*. }\end{array}$ \\
\hline & & & & & Fabric.\&c. \&c. & \\
\hline 426 & \begin{tabular}{|l|l|}
6 & 1539
\end{tabular} & I. ? & April, May..... & LLeo, Virgo ...... & $\begin{array}{r}\text { Chin.Rec.,Apian. } \\
\text { Ann. Aug.\&c. }\end{array}$ & \\
\hline 427 & 1545 & $\dddot{\sigma}$ & & & Aretius. & \\
\hline 428 & 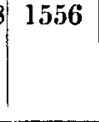 & $G$ & Feb.,Mar.,Apr. & $\begin{array}{r}\text { r. Libra, Virgo, } \\
\text { Bootes........ }\end{array}$ & $\begin{array}{r}\text { Annal. Aug., Car- } \\
\text { dan., Gemma. }\end{array}$ & $\begin{array}{l}\text { Elements com- } \\
\text { puted by } \\
\text { Halley*. }\end{array}$ \\
\hline
\end{tabular}

* See note, next page. 


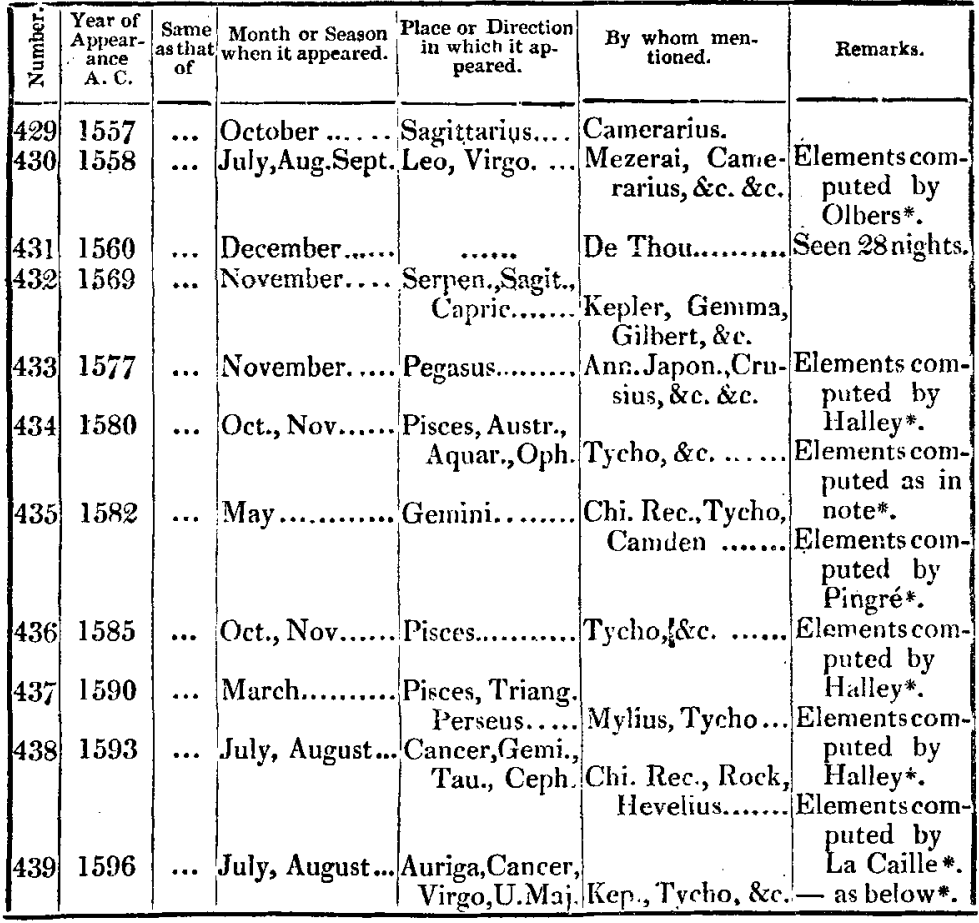

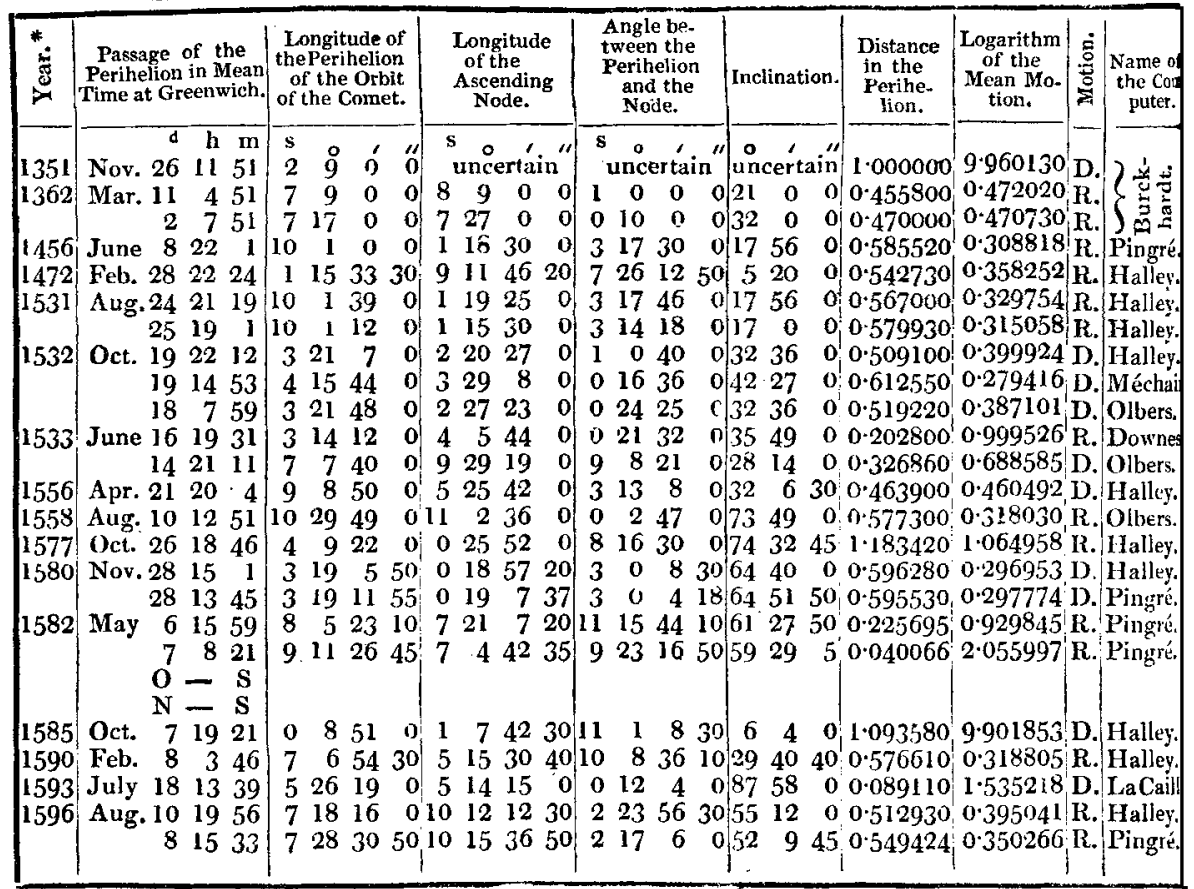

[To be continued.]

+ Eecentricity 0.967373.

ErRatcu in page 206 , line 8 from bottom, for $1264, \mathrm{G}$, read $\mathrm{G}$ ? 